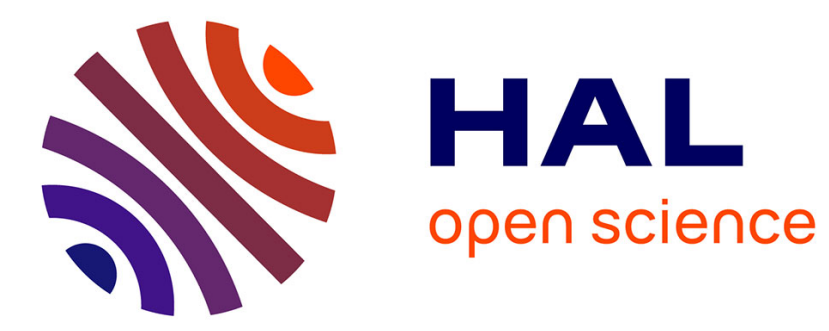

\title{
Perception des voyelles nasales du français par des apprenants hispanophones
}

David Alejandro Bustamante, Pierre Hallé, Claire Pillot-Loiseau

\section{To cite this version:}

David Alejandro Bustamante, Pierre Hallé, Claire Pillot-Loiseau. Perception des voyelles nasales du français par des apprenants hispanophones. XXXIIe Journées d'Études sur la Parole, Jun 2018, Aix-en-Provence, France. pp.603-611, 10.21437/JEP.2018-69 . halshs-02068353

\section{HAL Id: halshs-02068353 \\ https://shs.hal.science/halshs-02068353}

Submitted on 14 Mar 2019

HAL is a multi-disciplinary open access archive for the deposit and dissemination of scientific research documents, whether they are published or not. The documents may come from teaching and research institutions in France or abroad, or from public or private research centers.
L'archive ouverte pluridisciplinaire HAL, est destinée au dépôt et à la diffusion de documents scientifiques de niveau recherche, publiés ou non, émanant des établissements d'enseignement et de recherche français ou étrangers, des laboratoires publics ou privés. 


\title{
Perception des voyelles nasales du français par des apprenants hispanophones
}

\author{
David Alejandro Bustamante Pierre Hallé Claire Pillot-Loiseau \\ Laboratoire de Phonétique et Phonologie (LPP) UMR7018, CNRS-Paris3 / Sorbonne Nouvelle \\ 19 rue des Bernardins, 75005 Paris, France \\ david.alejandro.bustamante@gmail.com; pierre.halle@univ-paris3.fr \\ claire.pillotesorbonne-nouvelle.fr
}

\section{RESUME}

Notre objectif est d'examiner la perception des voyelles nasales françaises par des apprenants hispanophones d'Espagne et de Colombie de divers niveaux grâce à une série de tests de catégorisation et de discrimination. Les résultats de catégorisation des voyelles nasales du français (choix forcé parmi $/ \tilde{\varepsilon}, \tilde{a}, \tilde{\jmath} /$ ) dans des logatomes dissyllabiques dans les positions initiale, médiale

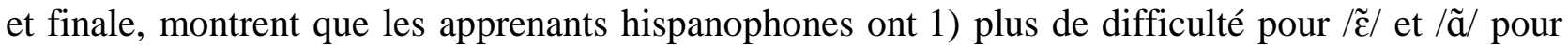

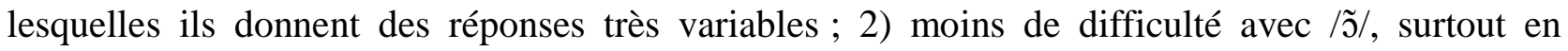
position finale (finale>médiale>initiale). Un test de catégorisation avec choix forcé entre les cinq catégories vocaliques de l'espagnol (/a, i, u, e, o/) permet de mieux comprendre comment les sujets hispanophones naïfs assimilent les voyelles nasales françaises. Nous proposons une modélisation quantitative, basée sur PAM (Best, 1995), prédisant les performances de discrimination à partir des assimilations et des notes de confiance subjective.

\section{ABSTRACT}

\section{Perception of Spanish-speaking learners of French nasal vowels}

The present study is intended to examine how Spanish-speaking learners of French from Spain and Colombia perceive the French nasal vowels according to level of fluency, using a series of categorization and discrimination. The results of the forced-choice within $/ \tilde{\varepsilon}$, ã, $\tilde{\jmath} /$ categorization tests on French nasal vowels appearing in two-syllable non-words in initial, medial, or final position show that Spanish-speaking learners encounter a greater difficulty with $/ \tilde{\varepsilon} /$ and /ã/, which receive quite variable responses; they encounter a lesser difficulty with $/ \tilde{\mathrm{J}} /$, especially in wordfinal position (final>medial>initial). A further categorization test requiring forced-choice responses within the five Spanish vowel categories (/a, i, u, e, o/) helped to better understand how Spanish-speaking listeners naive with respect to French assimilate French nasal vowels. We propose a quantitative modeling, based on PAM (Best, 1995), whereby discrimination performance is predicted from the observed assimilation and goodness-of-fit ratings.

MOTS-CLES : perception de L2, voyelles nasales du français, apprenants hispanophones espagnols et colombiens, sujets naïfs

KEY WORDS: L2 perception, French nasal vowels, Spanish and Colombian learners, naïve subjects 


\section{Introduction}

La prononciation d'une langue étrangère pose des difficultés d'apprentissage aux adultes: beaucoup de travaux se sont intéressés à ce sujet. Depuis la notion de "crible phonologique » (Troubetzkoy, 1938/1967), où le système phonologique de la langue maternelle agit comme un filtre influençant la perception des sons non natifs et par conséquent leur reproduction, d'autres travaux plus récents se sont attachés à rendre compte de ce phénomène concernant la perception des sons non-natifs par des auditeurs naïfs comme le Modèle d'assimilation perceptive (Perceptual Assimilation Model, PAM ; Best, 1995), ou par des apprenants d'une seconde langue (voir aussi PAM-L2 : Best \& Tyler, 2007). Selon le PAM, l'assimilation des sons d'une seconde langue (L2) à des catégories de la langue native (L1) se produit non seulement dans la perception des sons de manière isolée mais aussi dans la perception des contrastes de la L2. Un contraste de la L2 pouvant être assimilé à deux catégories différentes de la L1 (two category TC assimilation type) est mieux acquis qu'un contraste non-natif assimilé à une même catégorie de la L1 (single category SC assimilation type), ou bien un segment du contraste est acceptable et l'autre est déviant (category goodness CG assimilation type).

Le Modèle d'apprentissage de la parole (Speech Learning Model, SLM : Flege, 1995) représente une autre référence pour la perception des sons non-natifs mais il se concentre plus sur la production que la perception. D'autres travaux sur la perception de la L2 sont le Modèle magnétique de la langue native (Native Language Model, NLM: Kuhl, 2000), qui étudie notamment la perception de la parole chez des jeunes enfants à travers la notion d'aimants perceptifs constituant des prototypes des catégories des sons du langage; par rapport aux apprenants d'une L2, les sons non-natifs sont attirés par des aimants de la L1. Enfin, le Modèle de perception linguistique d'une seconde langue (Second Language Linguistic Perception Model, L2LP: Escudero, 2005), rend compte de l'influence que porte l'expérience de la L1 sur la perception et l'apprentissage d'une L2 par des sujets naïfs, des apprenants débutants à très avancés.

A notre connaissance, il existe très peu d'études sur la perception des voyelles nasales du français. Le travail d'Inceoglu (2014) aborde une perspective comparative de la perception des voyelles nasales du français après un entraînement multimodal (audio-visuel, visuel, et audio) auprès d'apprenants américains. Les effets de l'entraînement indiquent une progression de l'identification $([\tilde{\jmath}]>[\tilde{\varepsilon}]>[\tilde{a}])$ par rapport à l'expérience pré-entraînement, surtout pour $[\tilde{\varepsilon}]$, dans toutes les modalités soulignant la contribution des gestes visuels. Detey et al. (2015) présente les résultats d'une étude longitudinale sur le rapport entre perception et production des voyelles nasales du français par des apprenants japonais ; les résultats d'une expérience de discrimination montrent que l'opposition $/ \tilde{a} /-/ \tilde{\varepsilon} /$ est mieux perçue que l'opposition / $\tilde{a} /-/ \tilde{\partial} /$ par ces apprenants, mais que le taux de discrimination par voyelle est plus bas pour / $/$ / lorsqu'il est opposé à /ã/. Une autre étude sur la perception et la production des voyelles nasales auprès d'apprenants chypriotes hellénophones, montre la capacité des apprenants à bien distinguer le trait de nasalité du trait oral

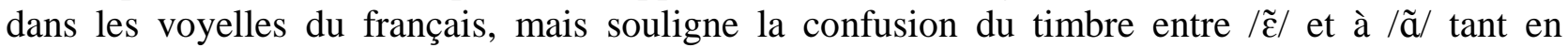
perception qu'en production (Kakoyianni-Doa et al. 2017). D'autres études portent sur la perception des voyelles nasales du français par des apprenants japonophones (Kamiyama, 2009), arabophones jordaniens (Nawafleh, 2013), et brésiliens (Desmeules-Trudel, 2013).

Par ailleurs, les recherches sur la perception des hispanophones concernant le système vocalique du français sont très limitées; nous trouvons seulement l'étude de Magnen et al. (2005) sur la perception de /i//y//u/ du français. Nous constatons qu'il n'y a pas de recherches sur la perception 
des voyelles nasales du français par des hispanophones. C'est pourquoi nous menons cette étude, mais aussi parce que d'autres travaux sur les voyelles nasales des hispanophones en production ont montré la difficulté qu'elles représentent pour l'apprentissage de la prononciation du français (Detey et al. 2010 ; Bustamante et al. 2014). En espagnol, le trait de nasalité existe pour les consonnes, mais il n'y a pas de voyelles nasales, seulement une nasalisation des voyelles par coarticulation.

L'objectif de cette étude est d'examiner la perception des voyelles nasales du français par deux groupes d'hispanophones: espagnols et colombiens. Le motif des deux populations d'hispanophones est d'étudier s'il y a une différence de traitement de la perception des voyelles nasales dans ces deux variétés de l'espagnol, tant par les apprenants que par les sujets naïfs du français. Nous avons effectué un premier test de catégorisation des voyelles nasales dans des nonmots à deux syllabes, afin d'observer si la position syllabique de la voyelle joue un rôle dans la perception. Sur la base des résultats de la première expérience, nous avons ensuite effectué un test de catégorisation des voyelles nasales selon les catégories vocaliques de l'espagnol avec des sujets espagnols et colombiens naïfs pour ce qui concerne le français pour observer les types d'assimilation des voyelles nasales. Finalement, basés sur les principes de PAM (Best, 1995), nous proposons une modélisation prédisant les performances de discrimination des voyelles nasales du français par les apprenants et les naïfs hispanophones.

\section{Matériel et méthode}

\subsection{Expériences et stimuli}

Nous avons mené trois types d'expériences pour étudier la perception des trois voyelles nasales françaises, / $\tilde{\varepsilon} /$, /ã/, / $/$ /, présentes dans la région métropolitaine de Paris, par les apprenants et les sujets naïfs hispanophones.

La première expérience a consisté en un test d'identification à choix forcé des voyelles nasales à choix forcé dans des non-mots à deux syllabes, test effectué auprès des apprenants espagnols et colombiens. L'intérêt d'utiliser des logatomes à deux syllabes est de nous permettre d'observer l'identification des voyelles nasales en fonction de trois positions syllabiques différentes, présentées séparément: position initiale absolue \#_CCV, position initiale post-consonantique CC_CV, et position finale CVC_\#. 108 stimuli ont été élaborés pour former les logatomes, produits par deux francophones natifs : un homme (54 stimuli), et une femme (54 stimuli) de la région Île-de-France. Le nombre des stimuli par sous-test, c'est-à-dire par position syllabique de la voyelle nasale est de 36. Le contexte phonétique des stimuli par sous-test est: [\#_pru]; [bl_tra, gl_se]; [claf_\#, vap_\#].

Ultérieurement, nous avons effectué un test d'identification des catégories phonétiques natives auprès de sujets hispanophones naïfs du français, avec le but d'observer les types d'assimilation que ces sujets réalisent des voyelles nasales dans leur système vocalique natif. Les stimuli critiques sont à 18 mots monosyllabiques du français portant une voyelle nasale. Pour le contexte

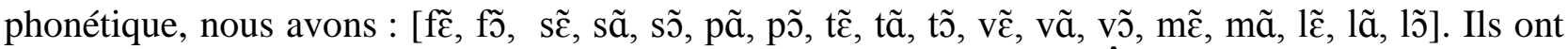
été produits par deux locuteurs francophones natifs de la région de l'Île-de-France (une femme et un homme), donc 36 stimuli au total. Une erreur technique ne nous a permis d'équilibrer le

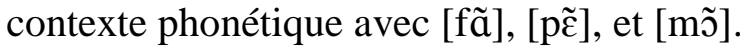


Enfin, les apprenants et les sujets naïfs ont passé un test de discrimination AXB sur les voyelles nasales du français. L'objectif de cette expérience est de prédire quelles sont les oppositions des voyelles nasales du français difficiles à discriminer par les auditeurs hispanophones. Douze mots monosyllabiques du français ont été utilisés pour former les stimuli du test de discrimination ; ils ont été produits trois fois par deux locuteurs (une femme et un homme de la région Ile-de-France) :

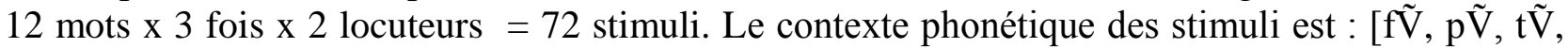
$\mathrm{k} \tilde{\mathrm{V}}, \mathrm{v} \tilde{\mathrm{V}}, 1 \tilde{\mathrm{V}}]$, où $\tilde{\mathrm{V}}$ est une des trois voyelles nasales.

\subsection{Participants}

30 apprenants espagnols (âge moyen 21.4 ; ET 5.2) et 26 apprenants colombiens (âge moyen 20.2 ; ET 2.3) ont participé à l'étude de perception. Nous les avons classés en fonction de leur niveau de français selon leur temps d'apprentissage et selon leur niveau dans l'établissement où ils apprennent le français : de six mois à un an d'apprentissage, les apprenants ont été classés comme débutants; de un an et demi à deux ans et demi, ils sont dans le niveau intermédiaire; et de trois ans et plus, ils sont dans le niveau avancé (Tableau 1). Les apprenants et les sujets naïs ont participé à cette étude depuis leur ville d'origine : à Bogota, pour les colombiens, et à Séville pour les espagnols. 18 francophones natifs $(16 \mathrm{~F}, 2 \mathrm{H})$ ont également participé en tant que groupe contrôle.

\begin{tabular}{|l|l|l|l|l|}
\cline { 2 - 5 } \multicolumn{1}{c|}{} & naïfs & débutants & intermédiaires & avancés \\
\hline Espagnols & $\mathbf{2 5}(21 \mathrm{~F}, 4 \mathrm{H})$ & $\mathbf{1 2}(10 \mathrm{~F}, 2 \mathrm{H})$ & $\mathbf{9}(6 \mathrm{~F}, 3 \mathrm{H})$ & $\mathbf{9}(5 \mathrm{~F}, 4 \mathrm{H})$ \\
\hline Colombiens & $\mathbf{1 9}(11 \mathrm{~F}, 8 \mathrm{H})$ & $\mathbf{1 0}(6 \mathrm{~F}, 4 \mathrm{H})$ & $\mathbf{9}(8 \mathrm{~F}, 1 \mathrm{H})$ & $\mathbf{7}(5 \mathrm{~F}, 2 \mathrm{H})$ \\
\hline
\end{tabular}

Tableau 1. Nombre des sujets naïfs, et des apprenants espagnols et colombiens selon leurs niveaux de français. F pour femme, $\mathrm{H}$ pour homme.

\subsection{Procédure}

Une phase d'entraînement pour chaque type de test a été proposée aux apprenants afin qu'ils se familiarisent avec le type de test, mais aussi pour qu'ils ce concentrent sur la position syllabique des voyelles nasales dans l'ensemble des sous-tests de catégorisation des voyelles nasales à choix forcé dans des logatomes. Les participants ont passé tout d'abord un sous-test d'identification des 36 logatomes contenant une voyelle nasale en position initiale absolue (\#_CCV), où ils devaient indiquer sur le clavier d'un ordinateur quelle était la voyelle nasale entendue dans cette syllabe : / $/$ /, /ã/, ou /õ/. Comme la plupart des apprenants n'étaient pas très familiarisés avec les symboles phonétiques, nous avons employé une transcription orthographique pour les réponses : 〈in〉, 〈an>, et $\langle$ on $\rangle$. Le principe était le même pour les autres sous-tests d'identification où la voyelle nasale se trouvait en position initiale post-consonantique (CC_CV), et en position finale (CVC_\#).

La catégorisation des voyelles nasales selon les 5 catégories vocaliques de l'espagnol a été passée par les apprenants et les sujets naïfs hispanophones. Après avoir entendu le stimulus, les auditeurs devaient signaler sur le clavier d'un ordinateur si le son vocalique entendu correspondait à l'une des voyelles de l'espagnol : /i/, /e/, /a/,/o/, /u/. Ensuite, sur une échelle de 1 à 5 , ils indiquaient le degré de bonne correspondance pour la catégorie attribuée à la voyelle nasale (5: bonne correspondance ; 1: mauvaise correspondance). Finalement, nous avons effectué un test de 
discrimination AXB des voyelles nasales. Après avoir entendu chaque triplet, le participant devait indiquer sur le clavier d'un ordinateur si le son correspondant à $\mathrm{X}$ était plutôt similaire au premier son $(\mathrm{A})$, ou plutôt au troisième son $(\mathrm{B})$.

\section{Résultats}

\subsection{Identification des voyelles nasales selon la position syllabique}

Les résultats d'identification des voyelles nasales ont été analysés en termes de taux d'identification correcte pour chaque population (colombiens, espagnols, et français) en fonction de la position syllabique des voyelles dans les logatomes. Les résultats montrent que, toutes voyelles et positions confondues, le groupe français présente le taux d'identification correcte le plus élevé (99\%), suivi du groupe espagnol (62\%), et en dernier du groupe colombien (53\%). La différence entre les trois groupes est significative, $F(2,7989)=698.2, p<0.0001$. Il y a aussi une interaction entre les effets Groupe et Voyelle nasale, $F(4,7983)=30.44, p<0.0001$, indiquant que l'identification des voyelles nasales est réalisée différemment selon les groupes. En effet, il n'y a pas de différence significative chez les français sur l'identification, $F(2,2049)=1.46, p=0.231$, mais il y a des différences significatives dans les groupes espagnol $(F(2,3237)=76.14, p<0.0001)$, et colombien $(F(2,2805)=82.5, p<0.0001)$.
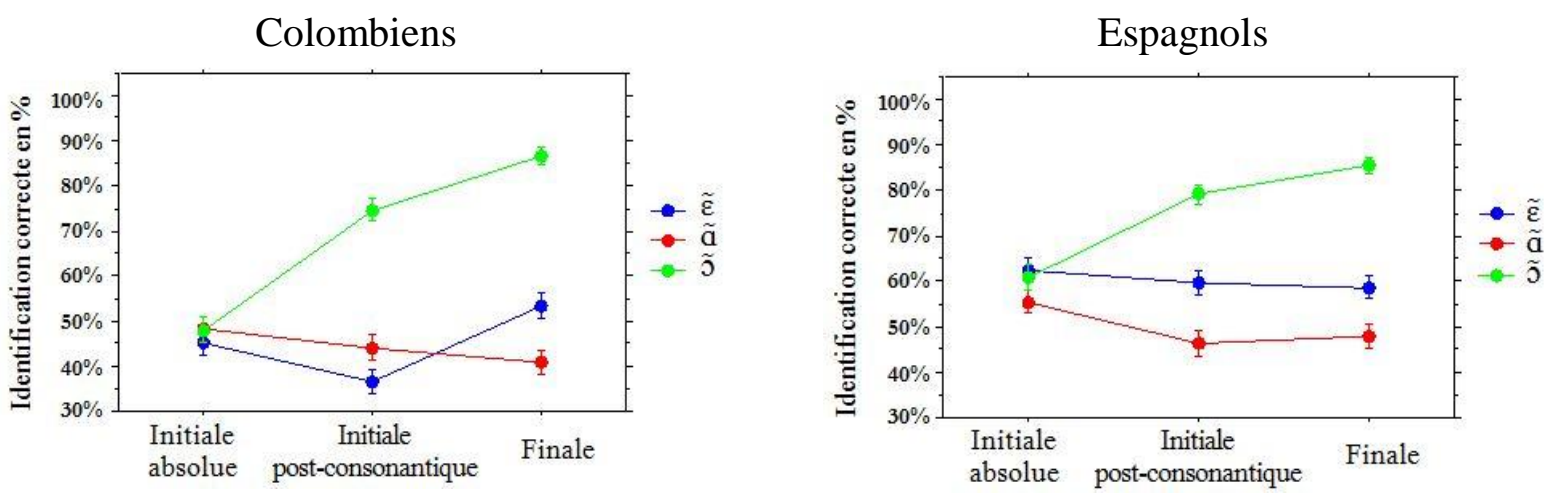

FIGURE 1. Taux moyen d'identification correcte (en pourcentages) des apprenants colombiens (à gauche) et espagnols (à droite) en fonction de la position syllabique des voyelles nasales dans le logatome : initiale absolue (\#_CCV), initiale post-consonantique (CC_CV), et finale (CVC_\#).

Les résultats, présentés dans la Figure 1, montrent un effet significatif concernant la position syllabique de la voyelle nasale dans le groupe colombien, $F(2,2805)=16.9, p<0.0001$, ce qui n'est pas le cas dans le groupe espagnol, $F(2,3237)=2.17 p=0.11$. Par contre, dans le groupe espagnol, on observe une différence significative sur l'identification entre les trois voyelles nasales $(/ \tilde{\mathrm{o}}>/ \tilde{\varepsilon} />/ \tilde{\mathrm{a}} /), F(2,3237)=76.14, p<0.0001$, alors que chez les Colombiens il y a aussi une différence significative $(/ \tilde{\partial} />/ \tilde{\varepsilon} /=/ \tilde{\mathrm{a}} /), F(2,2805)=82.5, p<0.0001$, sauf qu'il y a une confusion entre $/ \tilde{\varepsilon} /$ et $/ \tilde{\mathrm{a}} /$. L'influence de la position syllabique est déterminante pour la catégorisation de / $/$ /, commune aux deux groupes d'apprenants : il y a une progression de la position initiale absolue à la position finale. Comme le traitement perceptif de $/ \tilde{\varepsilon} /$ et $/ \tilde{a} /$ est plus difficile pour les apprenants, une variabilité d'identification est observée en fonction de la position syllabique. Nous allons observer la catégorisation que réalisent les sujets naïfs hispanophones des voyelles nasales du français selon les catégories vocaliques de l'espagnol. 


\subsection{Catégorisation des voyelles nasales selon les catégories de l'espagnol}

Les résultats, présentés dans la Figure 2, montrent la catégorisation des voyelles nasales que réalisent les hispanophones selon les catégories vocaliques de leur langue maternelle. Tout d'abord, nous observons les cas d'assimilation des sujets naïfs, car l'expérience linguistique du Français par les apprenants peut influencer leur catégorisation comme nous le verrons plus bas.
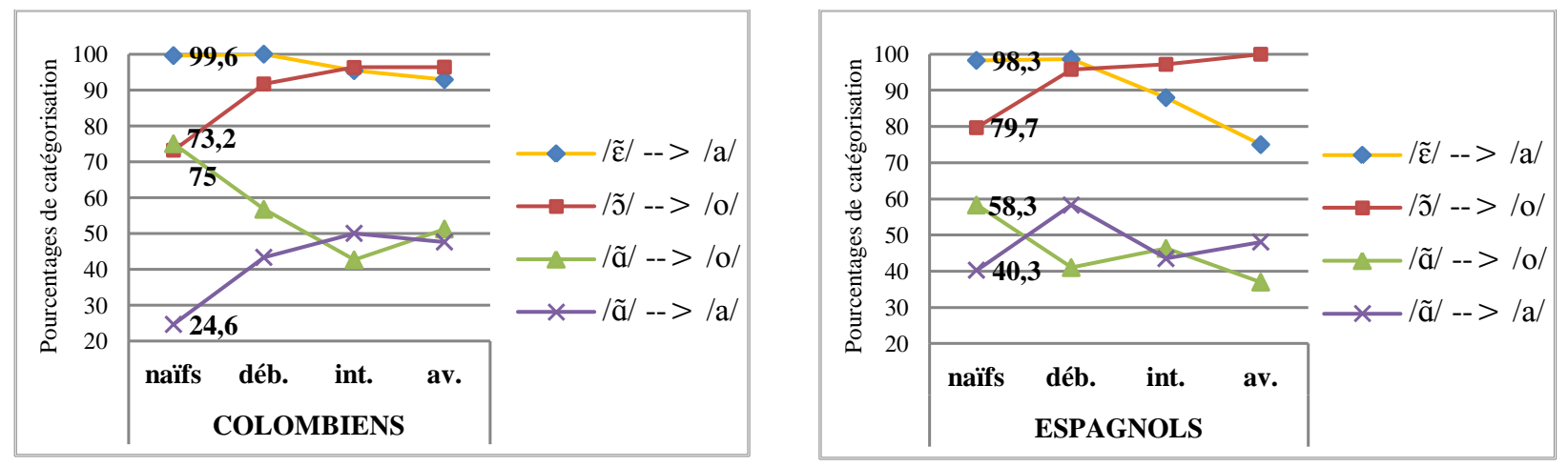

FIGURE 2. Assimilation en pourcentages des voyelles nasales du français / $\tilde{\varepsilon}$, ã, $\tilde{\jmath} /$, dans les catégories vocaliques de l'espagnol par les naïfs et les apprenants colombiens (gauche) et espagnols (droite).

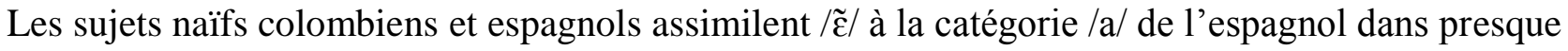
$100 \%$ des cas. Quant à la voyelle / $/$ /, elle est assimilée à /o/ dans $73.2 \%$ chez les colombiens, et un peu plus chez les espagnols, 79.7\%. Enfin, /ã/a été assimilé à deux catégories de l'espagnol : 75\% à $/ \mathrm{o} /$, et $24.6 \%$ à $/ \mathrm{a} /$ par les naïfs colombiens ; de la même manière les espagnols ont catégorisé /ã/ comme $/ \mathrm{o} /, 58.3 \%$ des cas, et comme /a/ dans $40.3 \%$.

Par ailleurs, la catégorisation des voyelles nasales en catégories vocaliques natives par les apprenants montre une assimilation influencée par leur expérience linguistique du français : / $/ \tilde{\varepsilon} /$ continue d'être assimilé à /a/, mais il y a un petit nombre de cas en /e/ (5.6\%), ainsi qu'une tendance à catégoriser sous l'influence d'une représentation de l'orthographe du français $(/ \tilde{\varepsilon} /=/ \mathrm{i} /$, 19.4\%). L'assimilation de / $/ \tilde{\mathrm{o}} / \mathrm{en} / \mathrm{o} / \mathrm{est}$ maintenue et augmentée chez les apprenants. Enfin, l'assimilation de / $\tilde{\mathbf{a}} /$ dans deux catégories de l'espagnol, /a/ et /o/, est maintenue, quoiqu'en moindre pourcentage, la double catégorisation est équilibrée : $/ \tilde{\mathrm{a}} /=/ \mathrm{a} /, 47.6 \%$, et $/ \tilde{\mathrm{a}} /=/ \mathrm{o} /, 51.2 \%$, chez les apprenants colombiens de niveau avancé ; alors que pour les espagnols de niveau avancé, l'assimilation de /ãa à /a/ représente $48.1 \%$, et pour /o/, 37\%. Nous observons donc, d'un côté, un affinement phonétique des voyelles nasales du français chez les apprenants, en fonction de l'assimilation en catégories vocaliques de l'espagnol, et d'autre part, une double catégorisation pour $/ \tilde{a} /:$ /a/ et /o/ de l'espagnol. L'intégration des résultats d'assimilation et des degrés de confiance est présentée ci-dessous.

\subsection{Modélisation des performances de discrimination des voyelles nasales}

Les résultats d'assimilation devraient permettent de formuler des prédictions sur les performances de discrimination des voyelles nasales par les hispanophones, à la lumière des prédictions du PAM sur la discrimination des contrastes non-natifs. Nous avons trouvé que $/ \tilde{\varepsilon} /$ est assimilé massivement à /a/ et / $/$ / à /o/, tandis que /ã/ est assimilé soit à /a/ soit à /o/ (/o/ étant dominant). Par conséquent, $/ \tilde{\varepsilon} /-/ \tilde{\partial} /$ est un contraste TC (two category assimilation : catégories /a/ et /o/) que PAM prédit bien 


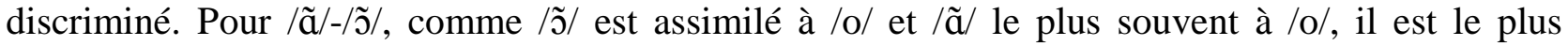
souvent SC (single category assimilation) ou CG (category goodness assimilation) que PAM

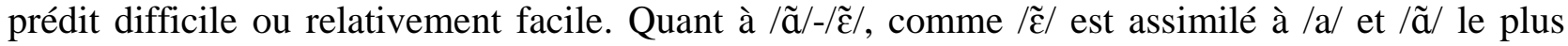
souvent à /o/, il est le plus souvent TC, donc facile, mais parfois SC, donc plus difficile, lorsque /ã/ est assimilé à /a/. Pour nos données d'assimilation, PAM prédirait donc la meilleure discrimination pour $/ \tilde{\varepsilon} /-/ \tilde{\partial} /$, suivi par $/ \tilde{a} /-/ \tilde{\varepsilon} /$, et la moins bonne pour $/ \tilde{\mathbf{a}} /-/ \tilde{\jmath} /$. C'est en effet ce que nous trouvons dans un test de discrimination que nous ne rapportons pas en détail ici (Figure 3). Pour affiner les prédictions, il s'agit de savoir dans quelle mesure un contraste est plutôt CG que SC. Typiquement, cette distinction est reflétée par des différences de rating (Best, 1995). Nous avons tenté de quantifier nos prédictions en prenant en compte les différences de rating. Si l'on ignore ces différences, une formule simple dérivant la performance de discrimination des données d'assimilation est $P(\neq /(\mathrm{x}, \mathrm{y}))=1-\sum_{i=1}^{5} P(\mathrm{ci} / \mathrm{x}) \times P(\mathrm{ci} / \mathrm{y}), c i$ étant les 5 catégories $/ \mathrm{a}, \mathrm{i}, \mathrm{e}, \mathrm{o}, \mathrm{u} /$. Le produit de probabilités correspond à la probabilité d'assimiler les deux termes du contraste, $\mathrm{x}$ et $\mathrm{y}$, à une même catégorie, donc de ne pas discriminer $\mathrm{x}$ et y sur cette catégorie. Notre idée est de pondérer ce terme par un facteur d'autant plus petit que la différence de rating est grande. La probabilité prédite de discriminer $\mathrm{x}$ et $\mathrm{y}, P(\neq /(\mathrm{x}, \mathrm{y}))$, est d'autant plus élevée que la différence est plus grande entre les ratings obtenus pour $\mathrm{x}$ et y sur la catégorie $c i$ pour i de 1 à 5 . Pour tester cette idée, nous avons utilisé une pondération très simple : $(4-|\operatorname{rating}(\mathrm{x}=c i)-\operatorname{rating}(\mathrm{y}=c i)|) / 4$. (Les ratings sont de 1 à 5, donc la plus grande différence est 4.). La Figure 3 montre les données de discrimination observées et prédites par notre "modélisation". Cette modélisation donne un poids aux différences de "category goodness", tout en restant basée sur les différences qualitatives entre les types de catégorisation prévues de PAM : TC versus SC ou CG. Nous nous proposons d'optimiser cette modélisation en utilisant une fonction paramétrable des différences de rating. Pour l'instant, nous ne montrons que la faisabilité de la modélisation.

Colombiens

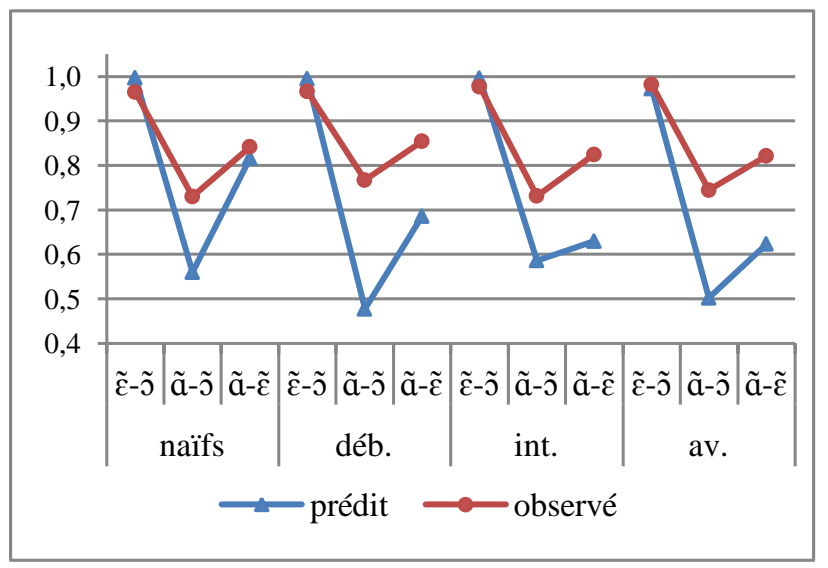

Espagnols

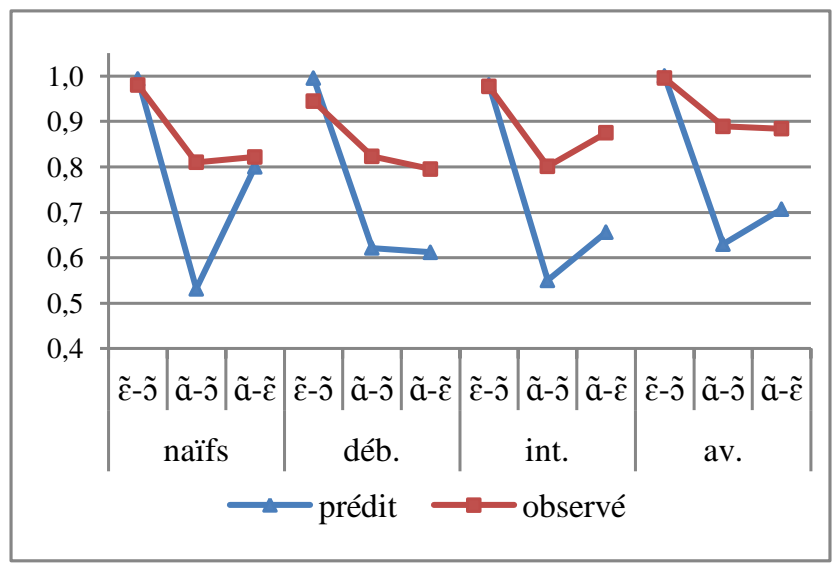

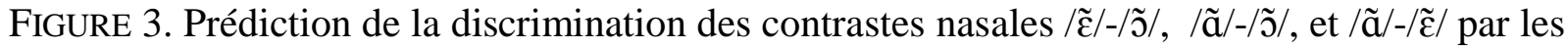
sujets naïfs et les apprenants hispanophones par niveaux de français : Colombiens, à gauche, et Espagnols, à droite ; prédiction en bleu et la discrimination observée en rouge.

Les résultats de discrimination, en rouge, montrent que le contraste / $/ \tilde{\varepsilon} /-/ \tilde{\partial} /$ est mieux discriminé par rapport aux autres contrastes dans les deux groupes d'hispanophones. Concernant le contraste /ã//̃̃/, il est le plus difficile à discriminer par les sujets colombiens, $F(2,3237)=120.6, p<0.0001$, comme prédit par le modèle quoique de manière plus amplifiée, alors que chez les espagnols les prédictions indiquent aussi une difficulté à discriminer l'opposition /ã/-/õ/ notamment, mais les résultats montrent qu'il n'y pas de différence significative de discrimination entre les deux 
contrastes $/ \tilde{\mathrm{a}} /-/ \tilde{\partial} /$ et $/ \tilde{\mathrm{a}} /-/ \tilde{\varepsilon} /(p=0.39)$, même s'il y a une différence significative entre tous les contrastes, $F(2,3957)=90.07, p<0.0001$.

\section{Discussion, conclusion et perspectives}

Cette étude cherchait à examiner la perception des voyelles nasales du français par les apprenants hispanophones dans un test d'identification en fonction de la position syllabique des voyelles nasales. Les résultats du test d'identification des voyelles nasales ont montré une confusion entre $/ \tilde{a} /-/ \tilde{\varepsilon} /$ chez les apprenants colombiens, et une difficulté plus accentuée, en particulier pour l'identification de /ã/ chez les espagnols. Même si l'identification de /õ/ est la moins problématique, elle est affectée par la position syllabique, notamment en position initiale absolue. Selon les difficultés observées, nous nous sommes intéressés à étudier comment les sujets naïfs hispanophones du français assimilent les voyelles nasales selon les catégories vocaliques de l'espagnol. Finalement, ces assimilations nous ont permis de formuler une série de prédictions des performances de discrimination des voyelles nasales par les apprenants et les naïfs hispanophones. La catégorisation des voyelles nasales que réalisent les sujets naïfs hispanophones selon les catégories de l'espagnol nous a permis de mieux comprendre les difficultés d'identification, ainsi que de proposer un modèle quantitatif de prédiction de la discrimination des voyelles nasales du français à partir des données de catégorisation et de rating. La double catégorisation de /ã/ en /a/ et $/ \mathrm{o} /$, dont le rating était plus important pour /o/, devient un facteur de difficulté pour les apprenants lorsqu'il est opposé à /õ/, et c'est ce que nous avons constaté avec les résultats de discrimination chez les colombiens. Quant aux sujets espagnols, les prédictions de discrimination n'ont coïncidé qu'avec les résultats de discrimination de $/ \tilde{\varepsilon} /-/ \tilde{\partial} /$; cependant leur difficulté avec l'identification de

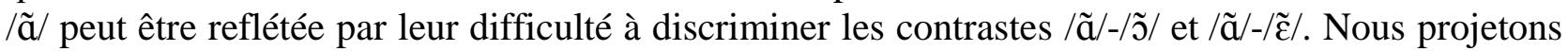
à l'avenir d'améliorer ce modèle de prédiction des performances de discrimination des voyelles nasales du français par des hispanophones. De plus, un rapport avec des résultats de production est à envisager.

\section{Remerciements}

Mille mercis aux personnes ayant participé à cette étude, aussi bien les sujets naïfs pour le français que les apprenants espagnols et colombiens. Merci aussi à M. Javier Enrique REDONDO, directeur du Département des Langues de la Pontificia Universidad Javeriana Bogota, Colombie, et à Mme Inmaculada ILLANES ORTEGA, directrice du Département de Philologie Française à l'Université de Séville, Espagne. Ce travail a bénéficié d'une aide du LabEx EFL en 2014 (ANR/CGI). Merci aussi à l'Université Paris Sorbonne-Cité pour la bourse de mobilité sortante 2015.

\section{Références bibliographiques}

BEST, C. T. (1995). "A direct realist perspective on cross-language speech perception," in Speech Perception and Linguistic Experience: Issues in Cross-language Research, Ed. W. Strange (Timonium, MD: York Press), 171-204.

BEst, C. T., TYLER, M. D. (2007). "Non native and second-language speech perception: commonalities and complementarities," in M. J. Munro \& O.S. Bohn (Eds.), Second Language Speech Learning: The Role of Language Experience in Speech Perception and Production, (pp. 13-34). Amsterdam: John Benjamins. 
BUSTAMANTE, D. A., AMELOT, A. et PILlOT-LOISEAU, C. (2014). «Étude de la production des voyelles nasales du français chez des apprenantes espagnoles et colombiennes », XXXe édition des Journées d'Études sur la Parole, Le Mans, 23 - 27 juin 2014, 576-580.

DESMEULES-TRUDEL, F. (2013). Perception des voyelles nasales du français québécois : aspects acoustiques et perceptifs. Mémoire de Maîtrise en Linguistique, Université de Laval, Québec.

DETEY, S., RACINE, I., KAWAGUCHI, Y., ZAY, F., \& BUEHLER, N. (2010). Évaluation des voyelles nasales en français en L2 en production : de la nécessité d'un corpus multitâches. In: Neveu, F., Durand, J., Klingler, T., Prévost S., Muni-Toké V. (éds.). Actes de CMLF'10 [CDROM], ILF, 1289-1301.

DETEY, S. et RACINE, I. (2015). Does perception precede production in the Initial stage of French nasal vowel quality acquisition by Japanese learners? A corpus-based discrimination experiment. Proceedings of ICPhS2015, Glasgow, 10-14 August.

ESCUDERO, P. (2005). "Linguistic perception and second language acquisition: Explaining the attainment of optimal phonological categorization," Ph.D. thesis, Utrecht University, Utrecht, Netherlands.

FLEGE, J. E. (1995). "Second language speech learning: Theory, findings and problems," in Speech Perception and Linguistic Experience: Issues inCross-language Speech Research, edited by W. Strange (York, Timonium, MD), pp. 233-272.

INCEOGLU, S. (2014). Effect of multimodal training on the perception of French nasal vowels. Concordia Working Papers in Applied linguistics, 5, 311-321.

KAKOYIANNI-Doa, F., MONVILLE-BURSTON, M., \& ARMOSTIS, S. (2017). "Les nasales $/ \tilde{\varepsilon} /$ et /ã / chez les apprenants hellénophones". Revue du Centre Européen d'Etudes Slaves - La revue | Numéro 6. [En ligne] Publié en ligne le 06 mars 2017. URL : http://etudesslaves.edel.univpoitiers.fr/index.php?id=1108 (consulté le 28/11/2017).

KAMIYAMA, T. (2009). Apprentissage phonétique des voyelles orales du français langue étrangère chez des apprenants japonophones. Thèse de doctorat. Université de la Sorbonne Nouvelle, Paris.

KUHL, P. K. (2000). A new view of language acquisition. Proceedings of the National Academy of SciencesUSA 97, 11850-11857.

MAGNEN, C., BILLIERES, M. \& GAILLARD, P. (2005). Surdité phonologique et catégorisation: perception des voyelles françaises par des hispanophones. Revue PArole, 33, 9-30.

NAWAFLEH, A. (2013). Difficultés de prononciation et de perception de voyelles du français par des apprenants arabophones: apprenants jordaniens. Presses Académiques Francophones, 535 pages.

TROUBETZKOY, N. S. (1938/1967). Principes de phonologie, Paris, Éditions Klincksieck. 